\title{
Improved Method for Point-Based Tracking
}

\author{
Andrei Danilchenko ${ }^{1}$, Andrew D. Wiles ${ }^{2,3}$, \\ Ramya Balachandran ${ }^{4}$, and J. Michael Fitzpatrick ${ }^{1}$ \\ ${ }^{1}$ Department of Electrical Engineering and Computer Science, Vanderbilt University, \\ Nashville, TN, USA \\ ${ }^{2}$ Northern Digital Inc., Waterloo, ON, Canada \\ ${ }^{3}$ Robarts Research Institute, The University of Western Ontario, London, ON, \\ Canada \\ ${ }^{4}$ Department of Otolaryngology, Vanderbilt University, Nashville, TN, USA
}

\begin{abstract}
Image-guided surgery systems have a wide range of applications where the level of accuracy required for each application varies from millimeters to low sub-millimeter range. In systems that use optical tracking, it is typical to use point-based registration without any weighting schemes to determine the pose of the tracked tool with very good accuracy. However, recent advancements in methods to estimate the measurement uncertainty for each tracked marker and the development of an anisotropically weighted point-based registration algorithm have allowed for the optical tracking accuracy to be improved. In this article, we demonstrate a new tracking method that improves the tracking accuracy by $20-45 \%$ over the traditional tracking methodology.
\end{abstract}

\section{Introduction}

Image-guided surgery (IGS) systems have improved the standard of care in brain, spine and orthopaedic interventions by combining pre-operative medical images and virtual reality using spatial tracking technologies [1. Recently, real-time imaging techniques, such as ultrasound and endoscopy, and robotics technology have been integrated with traditional IGS systems by tracking the imaging and robotic devices similarly to tracked surgical tools. The real-time images and robotic devices are displayed in the virtual reality environment resulting in an augmented view of the surgical target. One such application is the robotic drilling system for a mastoidectomy [2] where a section of bone is resected from behind the ear of a patient for various otolaryngology procedures. In this application, an optical tracking system (NDI Polaris Spectra ${ }^{\circledR}$, Waterloo, ON, Canada) is used to track the poses of both the patient and the robot. If the patient moves during the drilling procedure, the drilling plan is automatically updated to reflect the change in patient positioning using the real-time information provided by the optical tracking system. This procedure serves as an example of applications in which a high degree of tracking accuracy is crucial to success. Such applications motivate the focus of this paper, which is the development and validation of an improved method for highly accurate optical tracking.

T. Jiang et al. (Eds.): MICCAI 2010, Part III, LNCS 6363, pp. 587-594, 2010.

(C) Springer-Verlag Berlin Heidelberg 2010 
The method presented in this paper is the combination of two recently developed algorithms: (i) a method to estimate the anisotropic fiducial localizer error (FLE) at each of the optically tracked markers 3] and (ii) a registration procedure that accounts for the anisotropic weighting at each of the markers [4. We show that the FLE covariance statistics can be used as an anisotropic weighting function in the registration procedure. Accuracy improvements of $20-45 \%$ are demonstrated and this improvement is deemed important for applications that rely on highly accurate real-time tracking such as fully automatic robotic mastoidectomy.

\section{Method}

The goal of this paper is to demonstrate that the anisotropic weighted point-based registration algorithm [4] provides better results than the standard isotropic pointbased registration method $[5,6]$. The comparison is done using the target registration error (TRE) statistics for both registration methods.

\subsection{Computing the Real-Time Weightings}

The weighting for the anisotropic registration algorithm at each marker is determined using the FLE estimates from the algorithm presented in [3]. The FLE covariance matrix at a given marker $a$ is found by solving a complex set of linear equations that relates the FLE statistics at each marker to the estimated FRE statistics: $\boldsymbol{A}_{a} \boldsymbol{x}_{a}=\boldsymbol{b}_{a}$ where $\boldsymbol{A}_{a}$ is based on the geometry of the tracked rigid body, $\boldsymbol{x}_{a}$ is the six independent FLE covariance components and $\boldsymbol{b}_{a}$ is the six independent fiducial registration error (FRE) components estimated from the previous $M$ frames. For an optical tool with $N$ tracked markers, there are $N$ sets of linear equations that can be solved in order to obtain an estimate of the FLE at each marker. However, to improve numerical stability we can solve an overdetermined set of equations for a single FLE covariance matrix by stacking the matrices and vectors such that

$$
\boldsymbol{x}_{\text {avg }}=\left(\boldsymbol{A}_{\text {stack }}^{T} \boldsymbol{A}_{\text {stack }}\right) \boldsymbol{A}_{\text {stack }}^{T} \boldsymbol{b}_{\text {stack }}
$$

where

$$
\boldsymbol{A}_{\text {stack }}=\left[\begin{array}{c}
\boldsymbol{A}_{1} \\
\boldsymbol{A}_{2} \\
\vdots \\
\boldsymbol{A}_{N}
\end{array}\right] \text { and } \quad \boldsymbol{b}_{\text {stack }}=\left[\begin{array}{c}
\boldsymbol{b}_{1} \\
\boldsymbol{b}_{2} \\
\vdots \\
\boldsymbol{b}_{N}
\end{array}\right] \text {. }
$$

Taking the six independent FLE covariance components from $\boldsymbol{x}_{\text {avg }}$ (with or without stacking) and rewriting them as a FLE covariance matrix $\boldsymbol{\Sigma}$, the weightings are computed as

$$
\boldsymbol{W}=\boldsymbol{\Sigma}^{-1 / 2}
$$




\subsection{Obtaining a Good Estimate of the True Target Location}

In the work previously presented in the literature, the algorithms were tested using Monte Carlo simulations whereby the true location of the target was known exactly. However, experimentally the true target location is never known exactly and therefore a method of estimating the true target location is needed so that the TRE can be computed. Here we use two rigidly connected optically tracked tools shown in Fig. 1 to solve this problem.

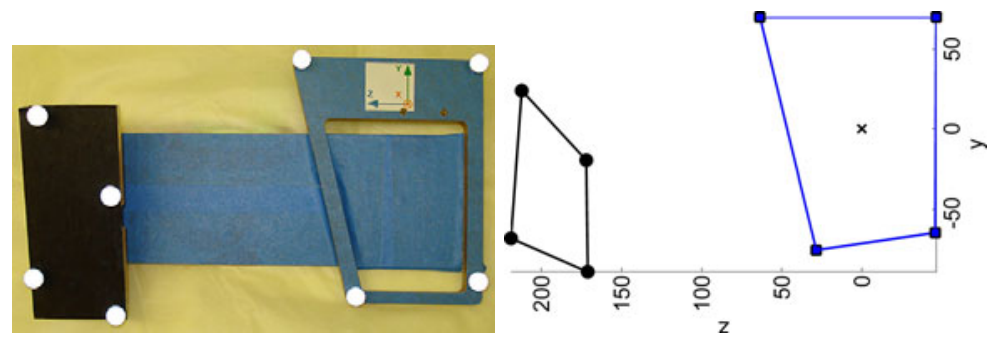

Fig. 1. Photograph and schematic of the tracked rigid bodies used in the experiment. The tracked tool under test is on the left and the tracked tool used to estimate the true location of the target is on the right. The target is the centroid of the right-hand tool, which is marked by an $\times$ in the schematic. Measurements are in millimeters and coordinate frame is the local tool coordinate frame.

In Fig. 1, the four markers on the left represent tracking markers rigidly attached to a tool (e.g., drill or hand-held pointer tool). The four markers on the right are arranged so that, for the robotic application, the centroid (denoted by $x$ in the schematic) is at the tip of the drill and for the hand-held-tool application the centroid is at the tip of the probe pointer. Previous to the tracking experiments a standard 'tool definition' calibration was carried out. The robotic arm was held stationary in several poses while the positions of the tool markers on the left and the target markers on the right were measured repeatedly (1000s of times). The average of these measurements over the various poses for each marker provides a highly accurate standard configuration. The centroid of the four markers on the right is defined to be the 'target' position (e.g., drill tip, or probe pointer tip). Then, during the tracking experiments (see Step iv below), each detected configuration of the tool is registered to the standard tool. Therefore, using the right-hand rigid body to estimate the true location of the target, the target is estimated with target localization error (TLE) statistics of

$$
\boldsymbol{\Sigma}_{\mathrm{tle}}=\frac{\boldsymbol{\Sigma}_{\mathrm{fle}, 1}+\boldsymbol{\Sigma}_{\mathrm{fle}, 2}+\ldots+\boldsymbol{\Sigma}_{\mathrm{fle}, N}}{N^{2}}, \quad \mathrm{RMS}_{\mathrm{tle}}=\sqrt{\operatorname{trace}\left(\boldsymbol{\Sigma}_{\mathrm{tle}}\right)}
$$

where $N$ is the number of markers on a tool ( $N=4$ for our example) and RMS is the root-mean-square statistic. If the FLE is homogeneous across the markers, then the covariance matrix and RMS reduce to $\boldsymbol{\Sigma}_{\text {tle }}=\boldsymbol{\Sigma}_{\mathrm{fle}} / N$ and $\mathrm{RMS}_{\text {tle }}=\mathrm{RMS}_{\mathrm{fle}} / \sqrt{N}$, respectively. 


\subsection{Comparing Isotropic and Anisotropic Registrations}

The TRE statistics of the isotropic and anisotropic registrations algorithms are compared using the centroid of the right-hand tracked tool as the target ground truth. The two rigid bodies are rigidly attached to one another and moved together. The experimental protocol is to carry out these steps for every frame of data returned from the tracking system:

(i) The 3D positions of each of the markers on both rigid bodies are measured with the optical tracking system at an instance in time which we will refer to as a frame of data.

(ii) The target location, $\boldsymbol{r}_{r e f}$, is obtained by taking the mean of the four markers on the right-hand tracked tool.

(iii) The right-hand tracked tool is registered using isotropic registration and the FLE estimates are updated for these markers. The TLE statistics of the target are obtained using (4).

(iv) The left-hand tracked tool is registered using isotropic registration and the FLE estimates are updated for these markers. The target location, $\boldsymbol{r}_{i s o}$, is computed using the transform computed from isotropic registration.

(v) The left-hand tracked tool is registered using anisotropic registration using the FLE estimates of the markers for the weighting as per (3). The target location, $\boldsymbol{r}_{\text {aniso }}$, is computed using the transform computed from anisotropic registration.

(vi) The TRE vectors for both registration methods are computed such that tre $\mathbf{e}_{i s o}=\boldsymbol{r}_{i s o}-\boldsymbol{r}_{\text {ref }}$ and tre $\mathbf{e}_{\text {aniso }}=\boldsymbol{r}_{\text {aniso }}-\boldsymbol{r}_{\text {ref }}$.

After 1000 frames of data are collected, the results are plotted and a set of observational statistics is computed. The results section provides a comparison of the algorithms for different dynamic paths over which the tool traveled and also provide examples of the FLE estimates for the markers obtained with the algorithm in [3].

\section{Results}

Using the method described in Section 2, we compared our new tracking method, performed using anisotropic registration 4, with traditional tracking method, performed using isotropic registration [5, 6]. The tracked tools were placed at a distance of approximately $160 \mathrm{~cm}$ from the tracking system. We noticed that the results of the comparisons are distinct for different types of motion, thus we collected data under the following conditions:

Test A: translate approximately $10 \mathrm{~cm}$ parallel to Polaris' x-axis (up-down) Test B: translate approximately $10 \mathrm{~cm}$ parallel to Polaris' y-axis (left-right) Test C: translate approximately $10 \mathrm{~cm}$ parallel to Polaris' z-axis (front-back) Test D: translate in all directions

Test E: rotate in all directions

Test F: random path including translations and rotations 
Table 1. Results of tests A-F. All statistics are computed over the usable 800 frames. The estimate of the TLE RMS is provided using (4). The TRE RMS statistics are computed for all the distance errors in a given test. The percent difference between the two methods is shown using the the isotropic RMS as the reference. Finally, noting that the TLE contributes to the measured TRE RMS statistic, see (5), we correct the TRE RMS statistics and recompute the TRE RMS percent difference.

\begin{tabular}{c|c|ccc|c}
\hline \hline Test & TLE RMS & TRE RMS & TRE RMS TRE RMS & TRE RMS \\
& & Isotropic & Anisotropic & \% Diff. & \% Diff Corr. \\
\hline A & $0.08 \mathrm{~mm}$ & $0.22 \mathrm{~mm}$ & $0.18 \mathrm{~mm}$ & $-19.3 \%$ & $-22.6 \%$ \\
B & $0.07 \mathrm{~mm}$ & $0.33 \mathrm{~mm}$ & $0.19 \mathrm{~mm}$ & $-42.4 \%$ & $-45.2 \%$ \\
C & $0.08 \mathrm{~mm}$ & $0.32 \mathrm{~mm}$ & $0.18 \mathrm{~mm}$ & $-42.8 \%$ & $-46.8 \%$ \\
D & $0.06 \mathrm{~mm}$ & $0.34 \mathrm{~mm}$ & $0.24 \mathrm{~mm}$ & $-28.7 \%$ & $-29.9 \%$ \\
E & $0.08 \mathrm{~mm}$ & $0.60 \mathrm{~mm}$ & $0.46 \mathrm{~mm}$ & $-23.2 \%$ & $-23.7 \%$ \\
F & $0.06 \mathrm{~mm}$ & $0.34 \mathrm{~mm}$ & $0.22 \mathrm{~mm}$ & $-34.5 \%$ & $-36.0 \%$ \\
\hline
\end{tabular}

A total of 1000 frames of data was collected for each test. Since a sliding window of 200 frames was used to estimate the FLE, only the last 800 frames are used for statistical analysis because it takes 200 frames until the FLE estimate stabilizes.

Three key sets of results are presented in Table 1. First, an estimate of the TLE RMS is given using (4) with estimates of the FLE covariance matrices found from the same FLE estimation algorithm used to determine the weightings for the anisotropic registration algorithm.

Next, the TRE RMS computed for both the isotropic and anisotropic registrations are provided. We notice that the anistropic TRE RMS is lower for each test and the percent difference between the two statistics is provided 1 .

Finally, we note that the TRE RMS for each registration has a contribution from the TLE where the measured TRE RMS can be related to the actual TRE RMS by

$$
\mathrm{RMS}_{\text {tre }, \text { meas }}^{2}=\mathrm{RMS}_{\text {tre }, \text { actual }}^{2}+\mathrm{RMS}_{\text {tle }}^{2} .
$$

Taking into consideration this relationship, we correct the percent differences between isotropic and anisotropic TRE RMS statistics by using the $\mathrm{RMS}_{\text {tre, actual }}$ and show this new percent difference in the last column of Table 11. A small increase in the accuracy is observed with this correction.

Moving beyond the observational statistics, we provide details of the data measured during Test F. In Fig. 2, the FLE RMS estimates are provided for (i) each marker estimated individually and (ii) the average FLE estimated by solving the over-determined system of equations in (11).

In Fig. 3, the magnitudes in the three principal directions of the average FLE covariance matrix are given. Here we observe the common behavior of optical tracking systems where one of the components is much larger than the other two directions. The direction of the higher magnitude error is along the viewing direction of the optical tracking system ( $z$-axis of the Polaris Spectra).

${ }^{1}$ The percent difference is computed using the isotropic registration as the reference value so that $\%$ Diff $=100 \times\left(\mathrm{RMS}_{\text {tre, } \text {, aniso }}-\mathrm{RMS}_{\text {tre }, \text { iso }}\right) / \mathrm{RMS}_{\text {tre }, \text { iso }}$. 


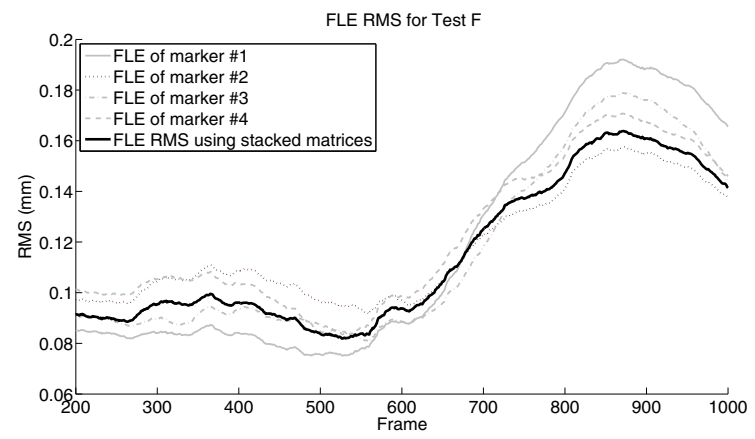

Fig. 2. Sample of the FLE RMS for Test F. The RMS is computed for (i) each marker individually and (ii) using the stacked, over-determined system of equations described in (11).

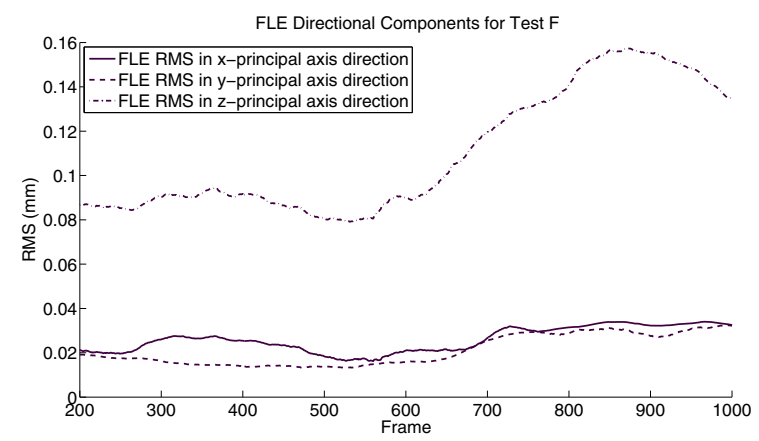

Fig. 3. Sample of the FLE directional components for Test F. The directional components are singular values of the covariance matrix where the principal axes of the covariance matrix are approximately aligned with the axes of the optical tracking system.

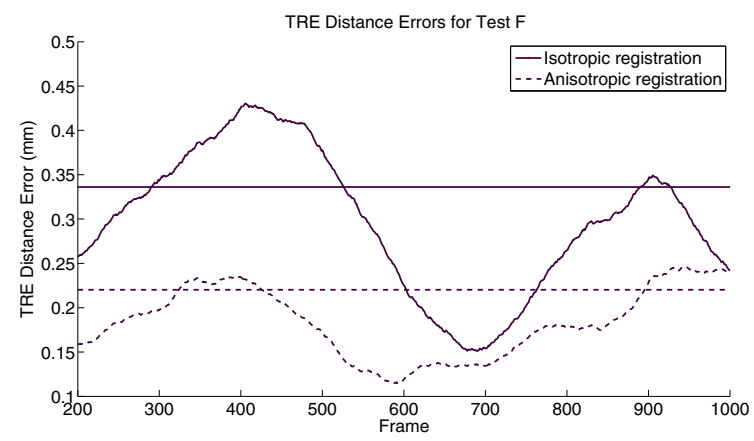

Fig. 4. TRE distance errors for Test F. The distance errors are smoothed using a moving average of 200 frames to better view the trend. Horizontal lines represent the RMS for each test. 
The last plot in Fig. 4 shows the TRE distance errors at each frame in Test F. We clearly demonstrate here that the anisotropic registration provides a better estimate of the target location. The TRE RMS computed over the entire data collection is shown with the horizontal lines. We also performed a paired t-test for this case and found $p \ll 0.05$, suggesting that the difference between isotropic and anisotropic TREs is statistically significant.

\section{Discussion}

We have developed an improved method for point-based tracking of rigid bodies. The improvement is accomplished by replacing the isotropic point-based registration method typically employed with one that takes into consideration the anisotropy of tracking system's FLE. Our method combines a recently published algorithm 3 for estimating the covariance matrix of anisotropic FLE with a novel rigid registration algorithm [4] that accommodates anisotropic weighting. Incorporating automatically generated weighting matrices optimized from the estimated FLE covariance matrices into the anisotropic registration algorithm exhibits a surprising level of accuracy, surpassing the current state of the art. Further improvements might be made by controlling for outliers or applying additional weightings during the solution of the equations, such as maximumlikelihood methods, M-estimators, regularization, etc. [7,8].

The method was tested on data obtained with NDI Polaris Spectra ${ }^{\circledR}$ with various motions of the tracked tool in the region approximately $160 \mathrm{~cm}$ from the tracking system. For a variety of motions, including pure translation, pure rotation, and combinations of both, we measured an increase in tracking accuracy in the range of $20-45 \%$, and in every case accuracy was improved. A statistical analysis confirmed that the tracking of optical rigid bodies using our new approach is more accurate than tracking using the standard method $(p \ll 0.05)$.

The input to each FLE calculation is a set of previous FRE vectors collected from the most recent $M$ frames, using the isotropic registration algorithm. We found that the size of the window, $M$, used for estimating the FLE statistics is an important factor in the tracking accuracy for our algorithm. The size of the window was investigated experimentally and we found that a contiguous collection of $M=200$ frames of FRE measurements produced the best results. Larger windows gave only a very small improvement in accuracy but also increased the lag in obtaining a good estimate of the FLE statistics. With a window size of $M=200$, one must allow the system to perform 200 data collections, before the improved tracking can commence. For the experiments described in this article, our method was implemented to run off-line in Matlab (Version 2009b, Mathworks, Inc., Natick, MA) on an Intel Core 2 Duo $2.2 \mathrm{GHz}$ with $2 \mathrm{~GB}$ of RAM while utilizing only one CPU. Each complete update, comprising FLE estimation and registration, required less than 6.4 milliseconds. For the NDI Polaris Spectra ${ }^{\circledR}$, whose update rate is $60 \mathrm{~Hz}$ (period of $17 \mathrm{~ms}$ ), our improved tracking algorithm can run in real-time. Furthermore, at $60 \mathrm{~Hz}$ with the NDI Polaris Spectra ${ }^{\circledR}$, a delay of only four seconds is required to obtain the first set of 200 
frames of FRE data to begin providing accurate estimates of the FLE statistics. In summary, the algorithm has promise to be included in imaged-guided surgery applications that use point-based registration for highly accurate optical tracking.

\section{Acknowledgements}

This work was supported by the NIH. Three authors (AD, RB, and JMF) gratefully acknowledge salary support via two grants-R01 DC008408 and R01 DC010184. The robot was purchased via R21EB002886.

\section{References}

1. Peters, T.M.: Image-guidance for surgical procedures. Physics in Medicine and Biology 51(14), R505-R540 (2006)

2. Danilchenko, A., Balachandran, R., Toennies, J.L., Baron, S., Munske, B., Wester III, R.J., Labadie, R.F.: Robotic mastoidectomy. In: Abstracts Selected for the American Otological Society Spring Meeting, Las Vegas, NV, USA (May 2010)

3. Wiles, A.D., Peters, T.M.: Real-time estimation of FLE statistics for 3D tracking with point-based registration. IEEE Transactions on Medical Imaging 28(9), 13841398 (2009)

4. Balachandran, R., Fitzpatrick, J.M.: Iterative solution for rigid-body point-based registration with anisotropic weighting. In: Miga, M.I., Wong, K.H. (eds.) Proceedings of SPIE, Medical Imaging, Visualization, Image-Guided Procedures, and Modeling, Orlando, FL, USA, vol. 7261, p. 72613D. SPIE, San Jose (February 2009)

5. Schönemann, P.H.: A generalized solution of the orthogonal procrustes problem. Psychometrika 31, 110 (1966)

6. Horn, B.K.P.: Closed-form solution of absolute orientation using unit quaternions. Journal of the Optical Society of America A 4(4), 629-642 (1987)

7. Huber, P.J.: Robust Statistical Procedures. SIAM, Philadelphia (1996)

8. Mair, E., Strobl, K.H., Suppa, M., Burschka, D.: Efficient camera-based pose estimation for real-time applications. In: The 2009 IEEE/RSJ International Conference on Intelligent Robots and Systems, pp. 2696-3703 (October 2009) 